\title{
Sustained local delivery of insulin for potential improvement of peri-implant bone formation in diabetes
}

\author{
HAN Yong ${ }^{1}$, ZHANG XueYan ${ }^{2}$, E LingLing ${ }^{1}$, WANG DongSheng ${ }^{1} \&$ LIU HongChen ${ }^{1 *}$ \\ ${ }^{1}$ Institute of Stomatology, Chinese General Hospital of PLA, Beijing 100853, China; \\ ${ }^{2}$ Chinese PLA 66400 Hospital, Beijing 100039, China
}

Received April 19, 2012; accepted June 5, 2012; published online October 22, 2012

\begin{abstract}
Dental implantation is an effective standard treatment modality to restore missing teeth and maxillofacial defects. However, in diabetics there is an increased risk for implant failure due to impaired peri-implant osseous healing. Early topical insulin treatment was recently shown to normalize diabetic bone healing by rectifying impairments in osteoblastic activities. In this study, insulin/poly(lactic-co-glycolic acid) (PLGA) microspheres were prepared by a double-emulsion solvent evaporation method. Microspheres were then incorporated in fibrin gel to develop a local drug delivery system for diabetic patients requiring implant treatment. In vitro release of insulin from fibrin gel loaded with these microspheres was assessed, and sustained prolonged insulin release over 21 days ascertained. To assess the bioactivity of released insulin and determine whether slow release might improve impaired diabetic bone formation, 3-(4,5-dimethylthiazol-2-yl)-2,5-diphenyltetrazolium bromide (MTT), alkaline phosphatase (ALP) activity, mineralized nodule formation, and ELISA (enzyme-linked immunosorbent assay) assays were performed. The insulin released from the drug delivery system stimulated cell growth in previously inhibited cells, and ameliorated the impaired bone-forming ability of human MG-63 cells under high glucose conditions. Fibrin gel loaded with insulin/PLGA microspheres shows potential for improving peri-implant bone formation in diabetic patients.
\end{abstract}

insulin, controlled release, peri-implant bone formation, diabetes, hyperglycemia, human MG-63 cells, osteoblastic activity

Citation: Han Y, Zhang X Y, E L L, et al. Sustained local delivery of insulin for potential improvement of peri-implant bone formation in diabetes. Sci China Life Sci, 2012, 55: 948-957, doi: 10.1007/s11427-012-4392-x

During the past decade, dental implant therapy has evolved into one of the major treatment modalities for the rehabilitation of missing or lost teeth, and maxillofacial defects with high predictability [1]. However, in patients with diabetes mellitus such implants are associated with a higher failure rate compared with the general population [2-4]. Diabetes mellitus is a metabolic disorder characterized by an increase in plasma glucose levels with a wide range of systemic complications, including myopathy, nephropathy, retinopathy, microvascular and macrovascular disease, altered wound and bone healing and periodontitis [5-8]. The asso-

*Corresponding author (email: Liuhongchen301@ @otmail.com) ciation between diabetes mellitus and impaired bone repair around endosseous implants and delayed osseointegration has been documented in experimental settings [9-11]. In addition to impaired biomechanical properties of the fracture callus, diabetes leads to reduced cellular proliferation, decreased bony content and inhibited mineralization in osseous healing $[12,13]$. This may contribute to retarded osseointegration of dental implants and increased implant failure rates in diabetic patients.

Insulin has been proposed as being an anabolic agent for bone [14], and appears to play a key role in regulating fracture healing $[12,15]$ and peri-implant bone repair [10] in diabetes mellitus. Studies have shown that insulin can in- 
fluence bone growth by directly binding to its receptors presented on osteoblasts $[16,17]$. With in vitro bone cell cultures, insulin has been shown to stimulate osteoblast proliferation, collagen synthesis, alkaline phosphatase production and mineralization $[16,18]$. In diabetic rats, insulin delivered at the fracture site could normalize cellular proliferation, chondrogenesis, mineralization, cartilage content and biomechanical properties of the fracture callus without affecting systemic blood glucose parameters [13]. Consequently, it is rational to develop a drug delivery system for local delivery of insulin to ameliorate impaired bone formation around implants, improve surgical osseointegration and enhance the success rates of dental implants in diabetic patients.

Poly(lactic-co-glycolic acid) (PLGA), a bioresorbable polymer with good biocompatibility, has been frequently utilized as a carrier material for sustained delivery of proteins or peptides $[19,20]$. Sustained-release formulations of insulin prepared with PLGA have been reported by some investigators as a potential treatment for type 1 diabetes mellitus [21,22]. Recently, Wang et al. encapsulated insulin inside PLGA microspheres and directly delivered the pulverized microspheres to the implant-bone interface in diabetic rats [23]. The implant-bone contact was improved by direct infiltration of insulin in the diabetic rats, but was still less than that of non-diabetic rats. Potentially, local administration of microspheres around the implants may display drug loss [24], which could reduce the active effect of the sustained-release drug on the osseointegration of dental implants. Other disadvantages, such as inconvenience during the operation, are also obstacles to clinical use of the local application of microspheres to improve osseointegration.

Fibrin gel, approved by the FDA for clinical use as a sealant, is a non-cytotoxic, fully-resorbable, biocompatible matrix and has been used as a carrier to deliver growth factors [25,26]. However, fibrin gel is not applicable for use as a long-term drug delivery system because of its insufficient duration of delivery [27]. Fibrin gels loaded with nanoparticles or nanospheres have been developed to achieve a more sustained release of bioactive agents than nanoparticles, nanospheres or fibrin gel alone [28,29]. Moreover, commercially available fibrin gel, which is a two-component system, is injectable. The gel can be delivered through a supplied applicator holding two liquid components in separate syringes, providing concurrent mixing and delivery. In addition, it can gel in situ within a few seconds of injection. Therefore, fibrin gel loaded with microspheres may act as a convenient drug delivery system for sustained release of insulin around implants.

We have established a novel drug delivery system by incorporating insulin/PLGA microspheres into fibrin gel. These have the potential to promote peri-implant bone formation in diabetes. Insulin/PLGA microspheres were prepared using the water-in-oil-in-water $(\mathrm{W} / \mathrm{O} / \mathrm{W})$ double- emulsion method. These microspheres were incorporated into fibrin gel and insulin release kinetics evaluated in vitro. Bioactivity and pharmacological properties of the released insulin were investigated in cultured cells.

\section{Materials and methods}

\subsection{Materials}

PLGA $(75: 25$, Mw $15 \mathrm{kD})$ was synthesized and kindly provided by the School of Life Science and Engineering, Southwest Jiaotong University (Chengdu, China). Bovine insulin and polyvinylalcohol (PVA) were obtained from Sigma (St. Louis, MO, USA), and insulin was used without further purification. A commercially available fibrin glue kit was purchased from Guangzhou Bioseal Biotech Co., Ltd. (Guangzhou, China). Minimum essential medium with Earle's balanced salts (MEM/EBSS) and heat-inactivated fetal bovine serum (FBS) were purchased from Hyclone (Logan, UT, USA). MEM nonessential amino acids (NEAA) were from Gibco (Grand Island, NV, USA). Trypsin from porcine pancreas, MTT and alizarin red $\mathrm{S}$ were from Sigma. Human collagen type I ELISA kit was from R\&D (Minneapolis, MN, USA). All other chemicals were of the highest grade commercially available.

\subsection{Preparation of insulin/PLGA microspheres}

Insulin/PLGA microspheres were prepared by the double-emulsion solvent evaporation method (W/O/W). Briefly, $3 \mathrm{mg}$ of lyophilized insulin powder was dissolved in $100 \mu \mathrm{L}$ $0.01 \mathrm{~N} \mathrm{HCl}$, and the $\mathrm{pH}$ adjusted to 4.0. Aqueous insulin solution $\left(100 \mu \mathrm{L}, 30 \mathrm{mg} \mathrm{mL}^{-1}\right)$ was mixed with $1 \mathrm{~mL}$ of dichloromethane (DCM) containing $100 \mathrm{mg}$ of PLGA, then ultra-sonicated (SCIENTZ-2D, SCIENTZ, Ningbo, China) at an energy output level of $20 \%$ in an ice bath for $2 \mathrm{~min}$. The primary emulsion was then re-emulsified with $10 \mathrm{~mL}$ of $1 \%$ PVA aqueous solution using a homogenizer (RW 20.n, IKA, Shanghai, China) at $1500 \mathrm{r} \mathrm{min}^{-1}$ for $5 \mathrm{~min}$. The $\mathrm{W} / \mathrm{O} / \mathrm{W}$ emulsion was poured into $10 \mathrm{~mL}$ of PVA aqueous solution and stirred at $500 \mathrm{r} \mathrm{min}^{-1}$ for $8 \mathrm{~h}$ at room temperature, allowing the DCM to evaporate. The emulsion gradually solidified as the solvent diffused from the emulsion droplets into the external phase. The resulting microspheres were washed and collected by filtration, sieving, centrifugation (TDZ5-WS, Xiangyi, Changsha, China) and lyophilization.

\subsection{Microsphere characterization}

The surface morphology of the insulin/PLGA microspheres was examined and photographed using a scanning electron microscope (S800, Hitachi, Tokyo, Japan). Samples were coated with gold palladium under vacuum using an ion-coater to achieve a film of $20 \mathrm{~nm}$ thickness. Particle size 
distribution was determined by laser diffractometry using a laser sizer (OMEC-15000, OMEC, Zhuhai, China).

\subsection{Determination of insulin loading and encapsulation efficiency of microspheres}

The amount of encapsulated insulin in the microspheres was determined after extraction with $\mathrm{CH}_{3} \mathrm{CN}$ and $0.01 \mathrm{~N} \mathrm{HCl}$ using HPLC. Samples $(20 \mu \mathrm{L})$ were injected into a chromatograph (LC-10AT VP, Shimadzu, Kyoto, Japan) equipped with an ultraviolet detector (SPD-10A VP, Shimadzu) and an Apollo C-18 column (150 mm $\times 4.6 \mathrm{~mm}$ internal diameter, $5 \mu \mathrm{m}, 100 \AA$ ) (Alltech, Deerfield, IL, USA). The mobile phase was a mixture of $27 \%$ acetonitrile and $73 \%$ buffer containing $0.2 \mathrm{~mol} \mathrm{~L}^{-1} \mathrm{NaHSO}_{4}$ adjusted to $\mathrm{pH}$ 2.3 with phosphoric acid. The flow rate was $1.0 \mathrm{~mL} \mathrm{~min}^{-1}$, with the wavelength set at $214 \mathrm{~nm}$. Insulin loading was expressed as percentage of amount of encapsulated insulin per unit dry weight of microspheres. The theoretical drug content was obtained under the assumption that the entire amount of drug was encapsulated with no drug loss at any stage of microsphere preparation. The encapsulation efficiency was calculated using the following formula: (measured insulin loading/theoretical insulin loading $) \times 100 \%$.

\subsection{Preparation of fibrin gel loaded with insulin/PLGA microspheres}

The fibrin glue kit was composed of lyophilized fibrinogen powder, lyophilized thrombin powder, potassium dihydrogen phosphate (KDP) solution and calcium chloride $\left(\mathrm{CaCl}_{2}\right)$ solution. Fibrin gel loaded with insulin/PLGA microspheres was prepared by dissolving lyophilized fibrinogen powder in $0.5 \mathrm{~mL}$ of KDP solution to obtain a mixture containing $12.5 \mathrm{mg}$ of fibrinogen. Lyophilized thrombin powder was dissolved in $0.5 \mathrm{~mL}$ of $40 \mathrm{mmol} \mathrm{L}^{-1} \mathrm{CaCl}_{2}$ solution, then 10 $\mathrm{mg}$ of insulin/PLGA microspheres was added to form a suspension containing $80 \mathrm{IU}$ of thrombin. Finally, the mixture and the suspension were loaded into the supplied applicator, which held the two components in separate syringes and provided simultaneous mixing and delivery, forming a fibrin clot on delivery.

\subsection{In vitro kinetics of insulin release}

Insulin release from PLGA microspheres was determined by suspending microspheres in $1 \mathrm{~mL}$ of phosphate buffer $(\mathrm{pH} 7.4)$ as $20 \%(\mathrm{w} / \mathrm{v})$ dispersion in a centrifuge tube. The tubes were placed in an incubator-shaker at $37^{\circ} \mathrm{C}$ and agitated at approximately $50 \mathrm{r} \mathrm{min}{ }^{-1}$. At designated sampling times $(1,3,5,7,10,14,18,21$ and $24 \mathrm{~d})$, the supernatant was completely withdrawn after centrifugation and replaced with fresh media. HPLC analysis of the supernatant was performed to determine the amount of insulin released from the microspheres.
In vitro release kinetics of insulin from fibrin clots containing insulin/PLGA microspheres or fresh insulin was determined as follows: samples $(1 \mathrm{~mL})$ containing $10 \mathrm{mg}$ of microspheres or the equivalent amount of fresh insulin as the encapsulated insulin were polymerized in centrifuge tubes containing $2 \mathrm{~mL}$ of phosphate buffer ( $\mathrm{pH} \mathrm{7.4).} \mathrm{The}$ samples were incubated at $37^{\circ} \mathrm{C}$ and agitated continuously. Supernatant was removed at prescribed times $(1,3,5,7,10$, 14, 18, 21 and $24 \mathrm{~d}$ ) and replenished with fresh media. The amount of insulin released was estimated using HPLC.

\subsection{Human MG-63 cell cultures}

Human MG-63 cells were kindly provided by the Tumor Research Institute of the Chinese Academy of Medical Science (Beijing, China). Cells were cultured in T75 flasks (Sigma-Aldrich, St. Louis, MO, USA) containing $10 \mathrm{~mL}$ of MEM/EBSS supplemented with NEAA and 10\% FBS at $37^{\circ} \mathrm{C} / 5 \% \quad \mathrm{CO}_{2}$. When the cells reached $80 \%-90 \%$ confluence, the medium was aspirated and cells were washed twice with $10 \mathrm{~mL}$ of PBS. Cells were then detached with 2 $\mathrm{mL}$ of $0.25 \%$ trypsin and EDTA solution, and incubated for $2 \mathrm{~min}$. Trypsin neutralization was carried out with $1 \mathrm{~mL}$ of FBS and $7 \mathrm{~mL}$ of PBS. Cells were then centrifuged (1500 r $\min ^{-1}, 5 \mathrm{~min}$ ) followed by resuspension in $10 \mathrm{~mL}$ of fresh media. Cells were counted by removing $10 \mu \mathrm{L}$ of cell suspension and combining it with an equal volume of trypan blue solution. This mixture was vortexed and a sample loaded into a hemocytometer chamber, with cells counted using an inverted microscope (DMIL, LEICA, Solms, Germany).

\subsection{Insulin bioactivity and the effect of insulin on os- teoblasts under high glucose}

\subsubsection{MTT assays}

The human MG-63 cell suspensions were diluted to 10000 cells $\mathrm{mL}^{-1}$ in MEM/EBSS containing $10 \% \mathrm{FBS}$ and seeded onto 96-well plates to a final well volume of $200 \mu \mathrm{L}(2000$ cells/well). After culturing for $24 \mathrm{~h}$, the cells were serum-starved overnight in serum-free MEM/EBSS. The medium was then exchanged, and cells treated with one of the following media: (a) MEM/EBSS containing NEAA, 5\% FBS and $5.5 \mathrm{mmol} \mathrm{L}^{-1}$ glucose (normal glucose, NG); (b) MEM/EBSS containing NEAA, 5\% FBS and $25 \mathrm{mmol} \mathrm{L}^{-1}$ glucose (high glucose, HG); (c) MEM/EBSS containing NEAA, 5\% FBS, $25 \mathrm{mmol} \mathrm{L}^{-1}$ glucose and $100 \mathrm{nmol} \mathrm{L}^{-1}$ fresh insulin; (d) MEM/EBSS containing NEAA, 5\% FBS, $25 \mathrm{mmol} \mathrm{L}^{-1}$ glucose and $100 \mathrm{nmol} \mathrm{L}^{-1}$ insulin released from fibrin gel loaded with insulin/PLGA microspheres; and (e) MEM/EBSS containing NEAA, 5\% FBS, $25 \mathrm{mmol}$ $\mathrm{L}^{-1}$ glucose and supernatant from fibrin gel loaded with placebo PLGA microspheres. Supernatant samples from release experiments of fibrin gel loaded with insulin/PLGA 
microspheres or placebo PLGA microspheres were taken on days 1, 7, 14 and 21, and diluted with MEM/EBSS containing NEAA. After culturing for $3 \mathrm{~d}, 20 \mu \mathrm{L}$ of MTT was added to each well and incubated for $4 \mathrm{~h}$ at $37^{\circ} \mathrm{C}$. Subsequently, the supernatants were removed; and the formazan crystals that had formed were solubilized with $150 \mu \mathrm{L}$ of dimethylsulfoxide. The absorbance value of each well was read at $490 \mathrm{~nm}$ using an ELx800uv reader (Bio-Tek, Winooski, VT, USA).

\subsubsection{ALP activity assay}

Cell suspensions were diluted to 200000 cells $\mathrm{mL}^{-1}$ in MEM/EBSS containing 10\% FBS and seeded onto 24-well plates to a final well volume of $1 \mathrm{~mL}$. The cells were serum-starved overnight and then incubated with the above-described media separately for $7 \mathrm{~d}$. Aliquots of supernatants from release experiments of the fibrin gel loaded with either insulin/PLGA microspheres or placebo PLGA microspheres were taken on days 1 and 5, and diluted with MEM/EBSS containing NEAA. Cells were initially incubated with media containing supernatant from day 1 for $4 \mathrm{~d}$. To this were then added media containing supernatant from day 5 , the whole being incubated a further $3 \mathrm{~d}$. On day 7 , culture supernatants were aspirated and ALP activity measured by an automatic biochemical analyzer (BS300, Mindray, Shenzhen, China).

\subsubsection{ELISA assay for the secretion of type I collagen}

Cells were treated as above and the culture supernatants were withdrawn on day 7 . The amount of type I collagen in the culture supernatant was determined using the human collagen type I ELISA kit according to the manual provided by the manufacturer.

\subsubsection{Assay of mineralized nodule formation}

Cells were seeded onto 24-well plates as above and serum-starved overnight. Subsequently, the cells were incubated with the five above-described media separately for 21 d. Supernatant samples were taken on days 1, 5, 7, 10, 14 and 18. Cultured cells were treated as above (ALP activity assay) for the first 7 days. Thereafter, at the corresponding time point the culture media were replaced with media-containing supernatant samples taken from the matching time point, i.e., the media-containing supernatant samples taken from day 7 of release experiments were added into plates on day 7 of cell culture and so on. The mineralized nodule formation was evaluated by alizarin red-S staining. Cells were rinsed twice with PBS followed by fixation with $95 \%$ ethanol for $15 \mathrm{~min}$ at room temperature. Cells were then stained with $1 \%$ alizarin red $\mathrm{S}(\mathrm{pH} 8.3)$ for $10 \mathrm{~min}$ at room temperature and rinsed three times with $50 \%$ ethanol. For quantification, the bound staining was eluted with $10 \%$ (w/v) cetylpyridinium chloride, and the absorbance value of the supernatants measured at $490 \mathrm{~nm}$ using an ELx800uv reader.

\subsection{Statistical analysis}

The software package SPSS 16.0 (Chicago, IL, USA) was used for statistical analysis. All data are presented as means \pm SD and $P<0.05$ was considered statistically significant. The results were evaluated by one way analysis of variance (ANOVA), with a least significant difference (LSD) test for post-hoc analyses. All experiments were repeated at least three times, and representative experiments are shown.

\section{Results}

\subsection{Surface morphology, particle size and encapsula- tion efficiency of microspheres}

Insulin/PLGA microspheres were a white powder. Under SEM, the microspheres were shown to be spherical with a smooth surface texture (Figure 1). The particle diameter of the insulin/PLGA microspheres was $(31.2 \pm 15.7) \mu \mathrm{m}$. Their encapsulation efficiency determined over three replicates was $61.1 \% \pm 4.3 \%$.

\subsection{In vitro kinetics}

Microspheres were incorporated into fibrin gel and in vitro release behaviors were investigated. The cumulative release rate of insulin from fibrin gel loaded with PLGA microspheres in PBS was determined using HPLC and compared with those from fibrin gel and insulin/PLGA microspheres (Figure 2). In these three systems, the fibrin gel exhibited the highest initial burst release of $62 \%$ and the shortest re-

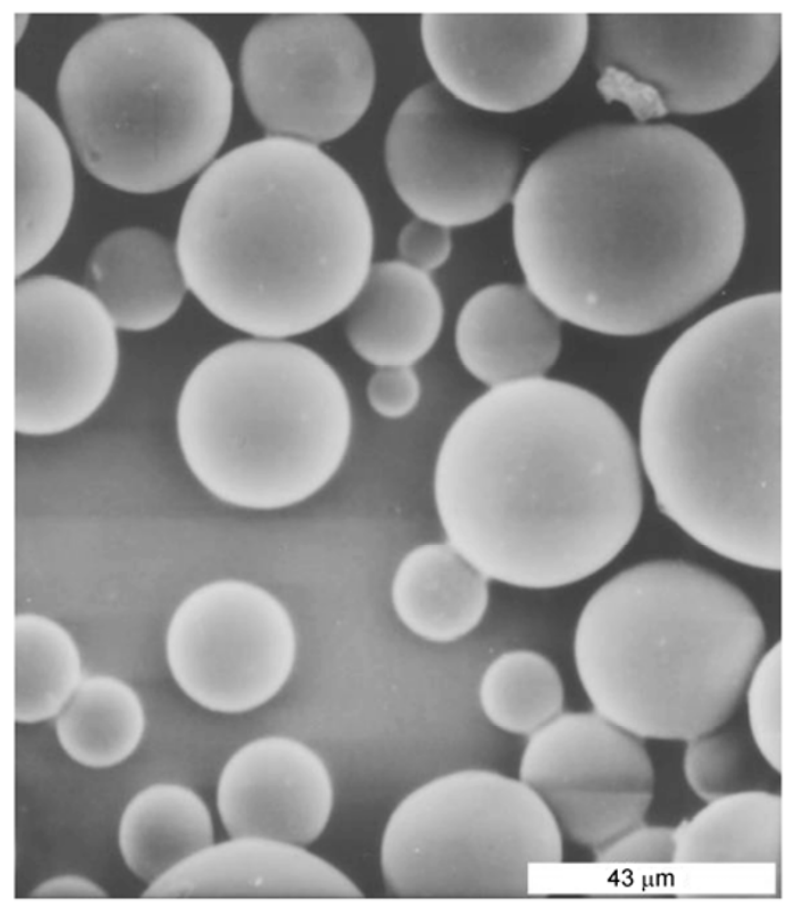

Figure 1 Morphology of insulin/PLGA microspheres by scanning electron microscopy. 


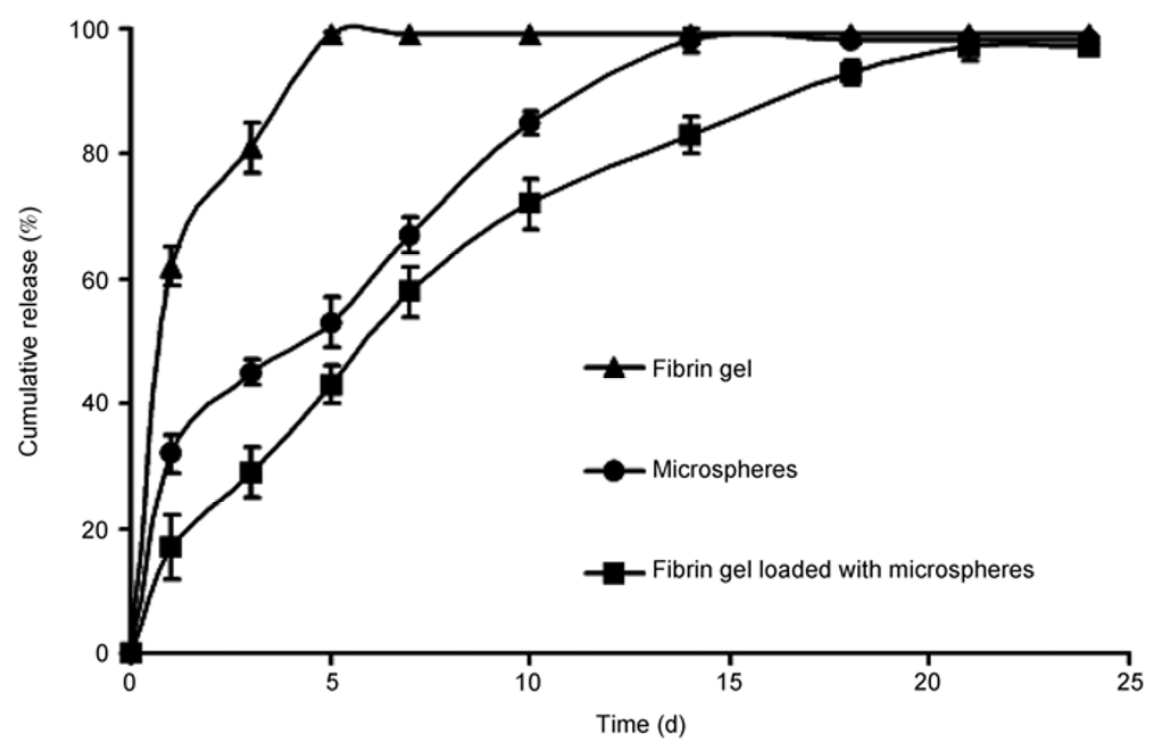

Figure 2 Profiles of insulin release from fibrin gel, microspheres and fibrin gel loaded with microspheres $(n=3)$.

lease length of $5 \mathrm{~d}$. In contrast, an insulin burst release from microspheres of approximately $32 \%$ was observed within the first $24 \mathrm{~h}$, followed by a slow release phase sustained for almost $14 \mathrm{~d}$. Compared with these two systems, the fibrin gel loaded with PLGA microspheres released insulin in a much steadier and more sustained manner. After incorporation of PLGA microspheres into fibrin gel, the initial burst release was decreased to $17 \%$ and insulin release was extended to $21 \mathrm{~d}$.

\subsection{Bioactivity and the effect of released insulin on os- teoblasts under high glucose}

Bioactivity of released insulin was determined by the MTT bioassay using quiescent MG-63 cells and compared with the activity of a fresh insulin control at the same concentration. As shown in Figure 3, high glucose induced a statistically significant reduction in the proliferation of MG-63 cells $(P<0.001)$. This inhibition was clearly reversed by the addition of either fresh or released insulin $(P<0.001)$. Released insulin from days 1 and 7 displayed high levels of bioactivity, similar to fresh insulin. However, a gradual decline in bioactivity of insulin was observed over the release period. Although released insulin on days $14(P<0.05)$ and $21(P<0.001)$ partly lost bioactivity, they still induced higher MG-63 cell proliferation which was statistically significant when compared with the placebo group and matched for collection time point $(P<0.001)$. Supernatants from the placebo group demonstrated no changes in MG-63 cell growth when compared with MG-63 cells under high glucose conditions without any treatment.

The ALP activity assay and ELISA to detect secretion of type I collagen were performed on human MG-63 cells to further investigate the bioactivity of released insulin and its effect on matrix development. Figure 4 shows that ALP activity was clearly decreased under high glucose conditions compared with normal glucose conditions $(P<0.001)$. ALP activity was increased significantly in MG-63 cells under high glucose conditions after insulin treatment compared with cells under high glucose conditions with no treatment $(P<0.001)$. ALP activity for the released insulin treatment group was statistically comparable to those for the group treated with fresh insulin and the normal glucose group. The increased ALP activity for the cells under high glucose conditions treated with released insulin was significantly higher than that of the placebo group $(P<0.001)$. Compared with the high glucose group receiving no treatment, no change was observed for the ALP activity of MG-63 cells after treatment with supernatants from the placebo group. The secretion of collagen type I by MG-63 cells cultured in different media is shown in Figure 5. The amount of collagen type I secreted by MG-63 cells under high glucose conditions without any treatment was significantly lower than that for the cells under normal glucose conditions $(P<0.01)$. After treatment of cells with high levels of glucose and insulin, collagen type I was significantly increased $(P<0.001)$, returning to a level equal to that of cells under normal glucose conditions. Additionally, there was no difference in the secretion of collagen type I between the cells treated with released insulin or fresh insulin. In contrast, MG-63 cells treated with supernatants from the placebo group resulted in no change in collagen type I secretion under high glucose pressure. Additionally, the amount of collagen type I from the cells treated with supernatants containing released insulin was significantly higher than those of the cells treated with supernatants containing no insulin, i.e., the placebo group $(P<0.01)$.

Calcium deposition was quantified using alizarin red $\mathrm{S}$ 


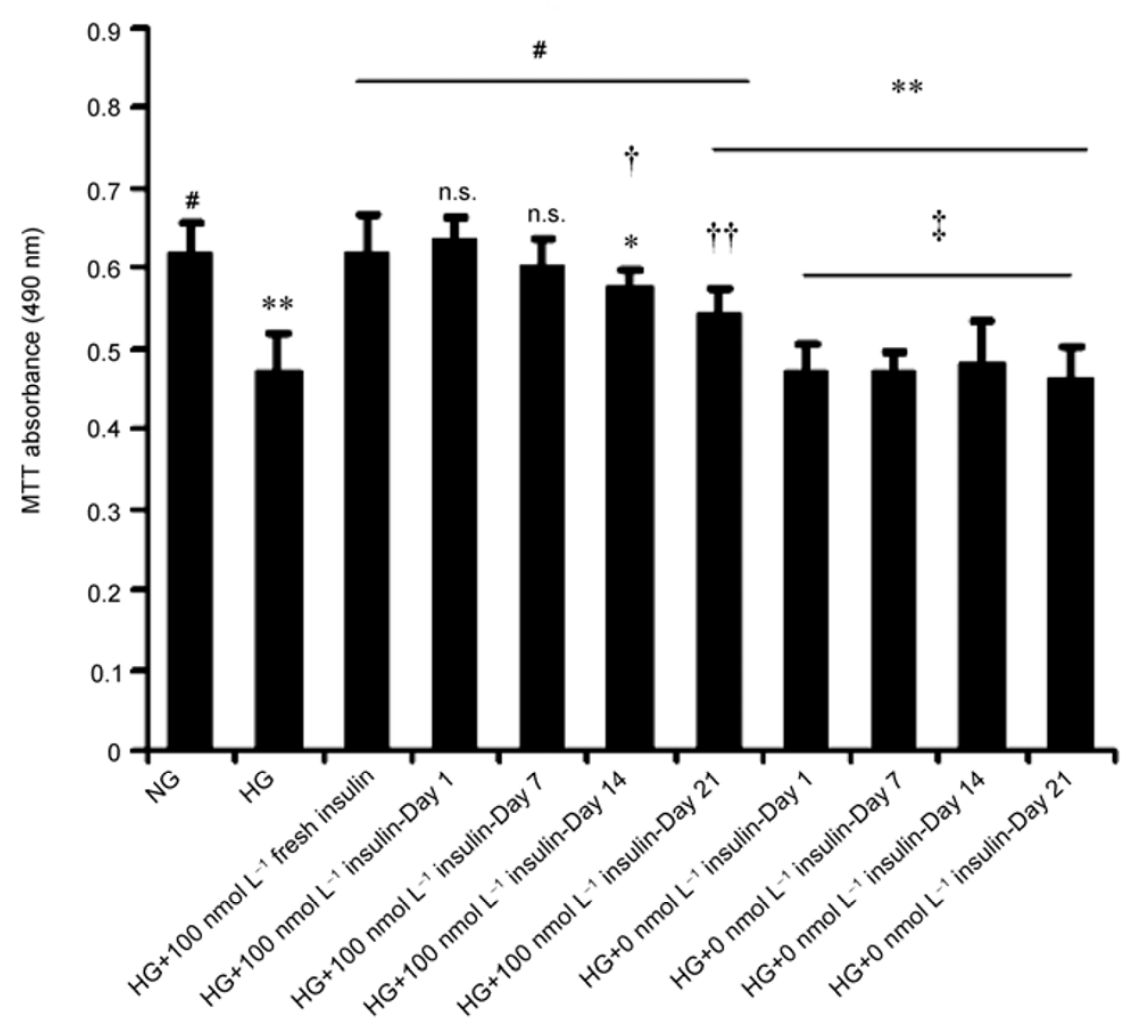

Figure 3 MTT bioassays to evaluate insulin bioactivity. The effect of insulin released from fibrin gel loaded with insulin/PLGA microspheres into supernatants sampled on days 1, 7, 14 and 21 in release experiments on osteoblast proliferation in human MG-63 cells under high glucose. Data represent the mean \pm SD, $n=8$ per group. *, $P<0.05$, **, $P<0.001$, compared to the normal glucose group; $\#, P<0.001$, compared to the high glucose group; n.s., not significant; $\dagger, P<0.05$, $\dagger, P<0.001$, released insulin compared to fresh insulin; $\dot{\ddagger}, P<0.001$, released insulin compared to placebo matched for the collection time point.

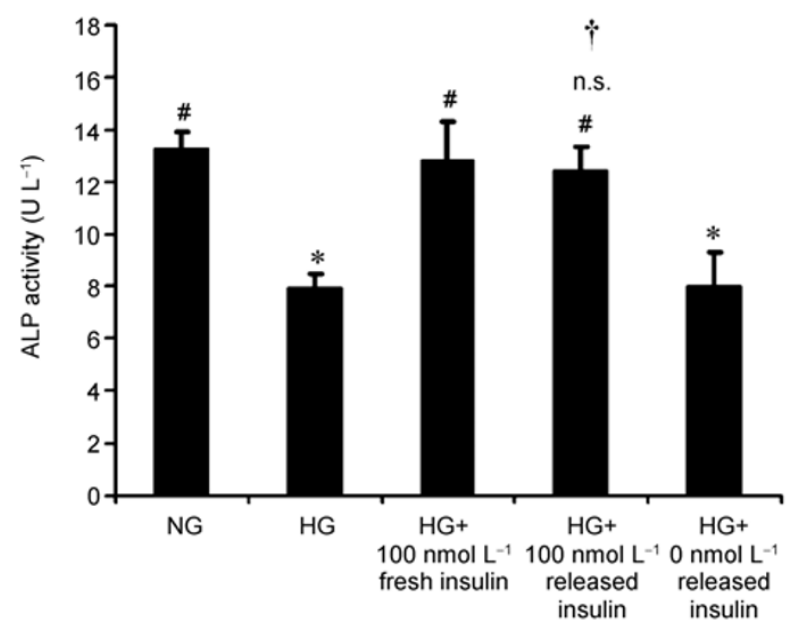

Figure 4 ALP activity assays to evaluate insulin bioactivity. The effect of insulin released from fibrin gel loaded with insulin/PLGA microspheres into supernatants in release experiments on early osteoblastic differentiation and bone forming ability of human MG-63 cells under high glucose. Data represent the mean $\pm \mathrm{SD}, n=6$ per group. $*, P<0.001$, compared to the normal glucose group; \#, $P<0.001$, compared to the high glucose group; n.s., not significant, released insulin compared to fresh insulin; $\uparrow, P<0.001$, released insulin compared to placebo.



Figure 5 ELISA assay for the secretion of type I collagen to evaluate insulin bioactivity. The effect of insulin released from fibrin gel loaded with insulin/PLGA microspheres into supernatants in release experiments on early osteoblastic differentiation and bone forming ability of human MG-63 cells under high glucose. Data represent the mean $\pm S D, n=6$ per group. * $P<0.05$, **, $P<0.01$, compared to the normal glucose group; \#, $P<0.01$, \#\#, $P<0.001$, compared to the high glucose group; n.s., not significant, released insulin compared to fresh insulin; $\dagger, P<0.01$, released insulin compared to placebo. 

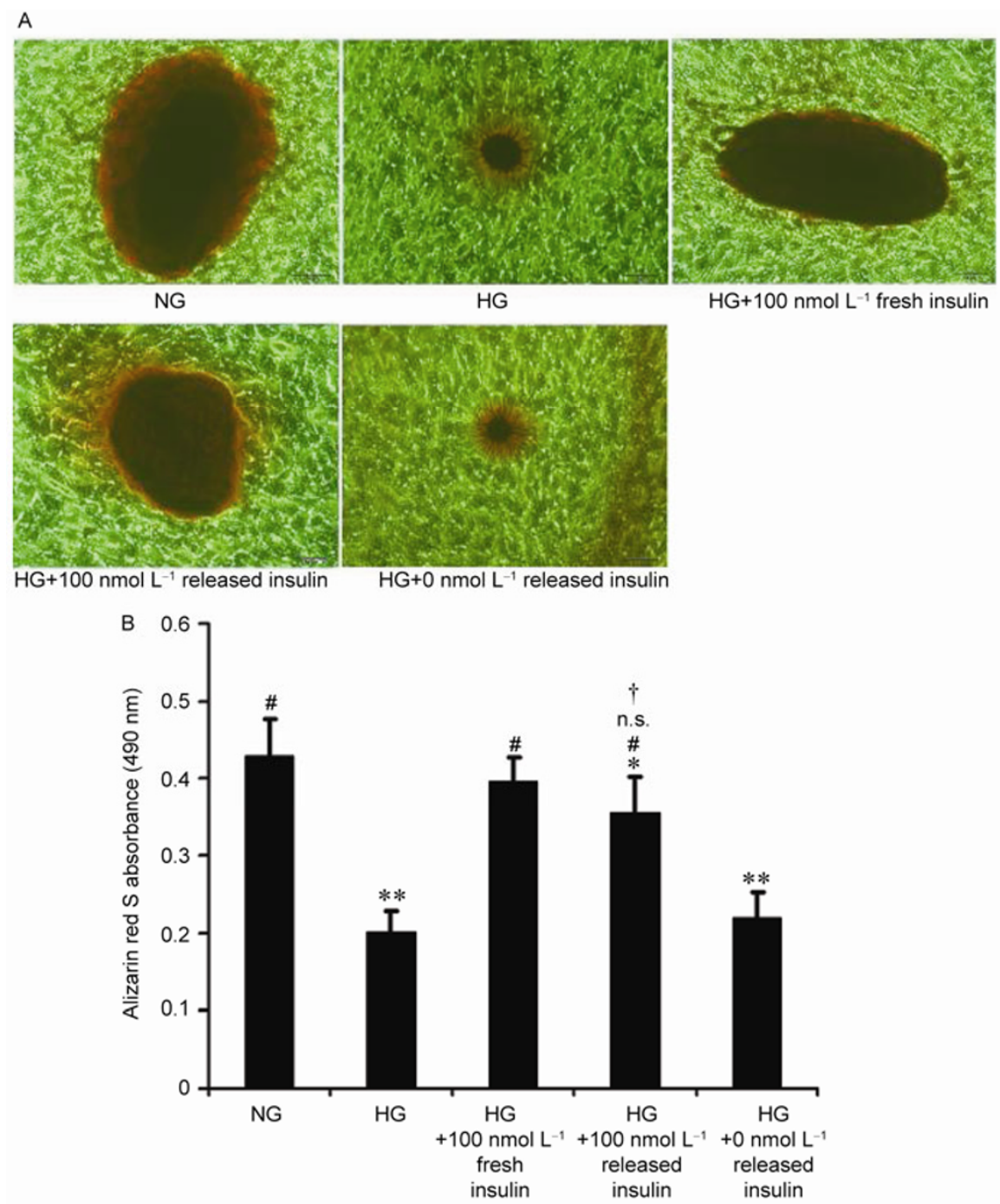

Figure 6 Assays of mineralized nodule formation to evaluate insulin bioactivity. A, Typical calcified nodules after alizarin red S staining. B, Calcium deposition quantified by alizarin red $\mathrm{S}$ absorbance. The effect of insulin released from fibrin gels loaded with insulin/PLGA microspheres into supernatants in release experiments on late osteoblastic differentiation and bone forming ability of human MG-63 cells under high glucose. Magnification, 200×. Data represent the mean $\pm \mathrm{SD}, n=6$ per group. $*, P<0.01$, ${ }^{*}, P<0.001$, compared to the normal glucose group; $\#, P<0.001$, compared to the high glucose group; n.s., not significant, released insulin compared to fresh insulin; $\dagger, P<0.001$, released insulin compared to placebo.

staining to investigate the bioactivity of released insulin and its effect on matrix mineralization. Typical calcified nodules are shown in Figure 6A. Quantitative measurements of alizarin red $\mathrm{S}$ absorbance showed that the normal glucose group displayed the highest mineralization level, whereas the high glucose and placebo groups exhibited the lowest (Figure 6B). A significant increase was observed in the alizarin red $\mathrm{S}$ absorbance on applying fresh insulin or released insulin to MG-63 cells under high glucose conditions compared with the high glucose group receiving no treatment $(P<0.001)$. Released insulin improved the mineralization by approximately the same degree as the fresh insulin. The mineralization of the cells treated with fresh insulin was statistically comparable with the normal level. However, mineralization of the cells treated with released insulin remained below the normal level $(P<0.01)$. No significant difference was ob- served in the mineralization level between the placebo group and the high glucose group without any treatment. The mineralization level of MG-63 cells treated with supernatants containing released insulin was significantly higher than that of cells treated with supernatants containing no insulin $(P<0.001)$.

\section{Discussion}

The success of implants relies on the degree of osseointegration [30]. This provides the anchoring of an implant due to the growth of bone around it. After implantation, the osseous healing around the implant is a relatively complicated proliferative, physiological process involving both bone formation and resorption. In the healing process, bone for- 
mation by osteoblasts is critical to osseointegration of an implant. However, in diabetes mellitus, bone formation is decreased, with suppressed osteoblast proliferation, differentiation and bone forming capacity during the critical early stage of osseous healing considered a plausible pathogenic mechanism [31]. One approach toward ameliorating impaired diabetic bone healing is through the topical application of insulin at fracture or implant sites $[13,23]$. In this study, we developed a drug delivery system for local insulin delivery around implants. First, we encapsulated insulin into PLGA microspheres using a W/O/W double-emulsion solvent extraction technique with an encapsulation efficiency of $61.1 \%$. The resulting microspheres appeared spherical and smooth, with a mean diameter of $31.2 \mu \mathrm{m}$. The microspheres were then incorporated into the fibrin gel, the release profile showing almost full release of insulin over $21 \mathrm{~d}$. The MTT bioassay, the ALP activity assay, an ELISA for the secretion of type I collagen and the mineralized nodule formation assay displayed high levels of sustained bioactivity for released insulin and a positive effect of released insulin on stimulating retarded diabetic osteoblastic activity.

Insulin can stimulate the growth of a number of cell types, including osteoblasts [18,32]. Moreover, insulin can act as an anabolic agent to mediate bone formation directly and/or indirectly [14]. After insulin binds to its specific high-affinity receptor, the insulin receptor, which is present on the surface of the osteoblast, activates downstream signaling cascades, such as the phosphatidylinositol 3-kinase and mitogen-activated protein kinase pathways to stimulate osteoblast proliferation and differentiation [18]. Insulin possesses primary, secondary and tertiary structure with labile bonds and side chains with chemically reactive groups. Disruption of these structures or modification of side chains readily occurs and can result in loss of insulin bioactivity [33]. Insulin was therefore encapsulated into PLGA microspheres to maintain bioactivity and thus to realize sustained local delivery of bioactive insulin when administered to the tissues around implants [23]. However, according to published research on the local application of PLGA microspheres around implants, the actual operation would be inconvenient and drug loss would occur during implantation. Wang et al. [23] reported that pulverized microspheres prepared in advance could be mixed with blood and then loaded on the implant surface before insertion into the surgically prepared implant osteotomy site. The osteotomy site should be drilled to a diameter a little smaller than that of the implant to achieve perfect bone to implant contact and sufficient implant primary stability, a prerequisite for efficient osseointegration [34]. Therefore, microspheres loaded on the implant surface would be scratched off due to mechanical friction during implant placement. Microspheres could also be administered into the tissue surrounding the dental implant after suspension in fresh blood until a clot was formed [24]. However, some of the microspheres might wash away during implant placement.
In our unpublished research, fibrin gel loaded with microspheres could be injected with a supplied applicator without any drug loss into an implant site properly prepared for a perfect bone-to-implant contact and consequent primary implant stability. The supplied applicator holds two components-a liquid mixture containing fibrinogen and a suspension containing thrombin and microspheres-in separate syringes and provides simultaneous mixing and delivery. After injection, this drug delivery mixture solidified into a semi-rigid gel matrix in situ within several seconds and maintained high flexibility for a short period. This ensured high initial retention of the drug around implants and sufficient time for implant placement. Therefore, fibrin gel loaded with microspheres may serve as a drug delivery system for local insulin delivery around implants, which would be more applicable to practical use than microspheres alone.

After insulin/PLGA microspheres were incorporated into fibrin gel, a more sustained and controlled release of insulin was achieved compared with either insulin/PLGA microspheres or fibrin gel alone. Fibrin gel loaded with insulin exhibited a short-term release of insulin with a high initial burst release, which may be accounted for by a simple diffusion-controlled mechanism. For PLGA microspheres, insulin was released more slowly and steadily. It was known that drug release from PLGA microspheres is a complicated process. This involves an initial burst release of surface-bound medication, the drug traveling through the interconnecting channels to the surface formed during microsphere hardening, and release due to physical or chemical erosion of the polymer. In contrast, the insulin release from fibrin gel loaded with PLGA/microspheres was further prolonged and stabilized by the combination of two individual drug delivery systems. This improvement in the release behavior of the newly developed drug delivery system could be explained by a combination of mechanisms of insulin release from microspheres and insulin diffusion through fibrin gel.

In our study, 5.5 and $25 \mathrm{mmol} \mathrm{L}^{-1}$ glucose were used to mimic the blood glucose in healthy individuals and in patients with poorly controlled diabetes, respectively. Our results clearly demonstrated profound inhibition in the proliferation, differentiation and bone forming capacity of osteoblast-like cells under high glucose conditions, which were consistent with previous reports [35-37]. A dose- and time-dependent mitogenic response of MG-63 cells to added insulin under high glucose conditions was confirmed in another study in our lab (unpublished data). Insulin bioactivity was tested by the MTT bioassay, which has the advantage of determining whether the released insulin preserves its bioactivity to induce a biological response in a functional biological system compared with other in vitro methods, such as HPLC and ELISA. In the present study, according to the MTT assay results, the released insulin collected during the whole release period significantly stimulated the proliferation of MG-63 cells under high glucose 
conditions. However, the released insulin exhibited a declining trend in the bioactivity of accelerating osteoblast proliferation over the release period. A similar phenomenon was reported by Hrynyk et al. [38]. The increasingly acidic microenvironment within degrading microspheres may contribute to the loss of insulin bioactivity [39]. Because insulin stability is critical for the drug delivery system, further studies will be initiated to enhance insulin stability by maintaining a near neutral microclimate $\mathrm{pH}$ within the degrading microsphere during release.

During bone formation, osteoblasts undergo a series of developmental stages, such as proliferation, matrix development and matrix mineralization [40]. ALP is expressed early in osteoblast development and type I collagen is the main component of the bone matrix. Thus ALP activity and the secretion of type I collagen could be used as indices to evaluate matrix development. Additionally, mineralized nodule formation can reflect matrix mineralization. For cells under high glucose conditions receiving no treatment, ALP production, synthesis of type I collagen and mineralized nodule formation were significantly decreased compared with cells cultured under normal glucose conditions. Our results were in accordance with previous studies [35-37]. The addition of released insulin to the culture media stimulated ALP production, type I collagen synthesis and mineralized nodule formation, with this high level of bioactivity similar to that for fresh insulin. Therefore, while high glucose greatly disrupted the development of osteoblasts and retarded osteoblastic activity, having deleterious effects on osteoblast differentiation and bone forming activity, the continuously released bioactive insulin attenuated these effects. Taking these results together with those from the MTT bioassay, we demonstrated the direct bioactivity of released insulin and indicated the potential for the local application of fibrin gel loaded with insulin/PLGA microspheres to improve impaired bone formation around implants in diabetes mellitus.

Supernatants from placebo groups were also assayed to identify any potential biological response to degradation products. Supernatants containing released insulin significantly stimulated cellular proliferation, promoted ALP activity, increased type I collagen synthesis and enhanced calcium deposition in human MG-63 cells under high glucose conditions when compared with supernatants from the placebo group. Additionally, no significant differences were observed between the placebo group and the high glucose group receiving no treatment. These data confirmed the bioactivity of released insulin and demonstrated that degradation products had no stimulatory effects.

The above results suggest that fibrin gel loaded with insulin/PLGA microspheres offers great potential to act as a drug delivery system for sustained delivery of bioactive insulin around dental implants with practical convenience. The limited results of this study still allow us to speculate that insulin released from the fibrin gel loaded with PLGA microspheres may ameliorate impaired peri-implant bone formation in diabetes, and thus improve compromised osseointegration. This study justifies continued research in diabetic animal models to further investigate the effect of sustained insulin delivery via fibrin gels loaded with insulin/PLGA microspheres around implants on improving peri-implant bone formation and osseointegration in diabetes.

1 Pjetursson B E, Bragger U, Lang N P, et al. Comparison of survival and complication rates of tooth-supported fixed dental prostheses (FDPs) and implant-supported FDPs and single crowns (SCs). Clin Oral Implants Res, 2007, 18: 97-113

2 Morris H F, Ochi S, Winkler S. Implant survival in patients with type 2 diabetes: placement to 36 months. Ann Periodontol, 2000, 5: 157-165

3 Moy P K, Medina D, Shetty V, et al. Dental implant failure rates and associated risk factors. Int J Oral Maxillofac Implants, 2005, 20: 569-577

4 Zupnik J, Kim S W, Ravens D, et al. Factors associated with dental implant survival: a 4-year retrospective analysis. J Periodontol, 2011, 82: 1390-1395

5 Eastman R C, Vinicor F. Report of the expert committee on the diagnosis and classification of diabetes mellitus. Diabetes Care, 1997, 20: 1183-1197

6 Taylor G W, Burt B A, Becker M P, et al. Non-insulin dependent diabetes mellitus and alveolar bone loss progression over 2 years. J Periodontol, 1998, 69: 76-83

7 Lalla E, Lamster I B, Drury S, et al. Hyperglycemia, glycoxidation and receptor for advanced glycation endproducts: potential mechanisms underlying diabetic complications, including diabetes-associated periodontitis. Periodontol, 2000, 23: 50-62

8 Kaur G, Holtfreter B, Rathmann W, et al. Association between type 1 and type 2 diabetes with periodontal disease and tooth loss. J Clin Periodontol, 2009, 36: 765-774

9 Hasegawa H, Ozawa S, Hashimoto K, et al. Type 2 diabetes impairs implant osseointegration capacity in rats. Int $\mathrm{J}$ Oral Maxillofac Implants, 2008, 23: 237-246

10 Siqueira J T, Cavalher-Machado S C, Arana-Chavez V E, et al. Bone formation around titanium implants in the rat tibia: role of insulin. Implant Dent, 2003, 12: 242-251

11 Takeshita F, Murai K, Iyama S, et al. Uncontrolled diabetes hinders bone formation around titanium implants in rat tibiae. A light and fluorescence microscopy, and image processing study. J Periodontol, 1998, 69: 314-320

12 Beam H A, Parsons J R, Lin S S. The effects of blood glucose control upon fracture healing in the BB Wistar rat with diabetes mellitus. J Orthop Res, 2002, 20: 1210-1216

13 Gandhi A, Beam H A, O'Connor J P, et al. The effects of local insulin delivery on diabetic fracture healing. Bone, 2005, 37: 482-490

14 Thrailkill K M, Lumpkin C K Jr., Bunn R C, et al. Is insulin an anabolic agent in bone? Dissecting the diabetic bone for clues. Am J Physiol Endocrinol Metab, 2005, 289: E735-745

15 Hough S, Avioli L V, Bergfeld M A, et al. Correction of abnormal bone and mineral metabolism in chronic streptozotocin-induced diabetes mellitus in the rat by insulin therapy. Endocrinology, 1981, 108: 2228-2234

16 Pun K K, Lau P, Ho P W. The characterization, regulation, and function of insulin receptors on osteoblast-like clonal osteosarcoma cell line. J Bone Miner Res, 1989, 4: 853-862

17 Zhang W, Shen X, Wan C, et al. Effects of insulin and insulin-like growth factor 1 on osteoblast proliferation and differentiation: differential signalling via Akt and ERK. Cell Biochem Funct, 2012, 30: 297-302

18 Yang J, Zhang X, Wang W, et al. Insulin stimulates osteoblast proliferation and differentiation through ERK and PI3K in MG-63 
cells. Cell Biochem Funct, 2010, 28: 334-341

19 Liu B, Dong Q, Wang M, et al. Preparation, characterization, and pharmacodynamics of exenatide-loaded poly(DL-lactic-co-glycolic acid) microspheres. Chem Pharm Bull, 2010, 58: 1474-1479

20 Zhang J X, Zhu K J, Chen D. Preparation of bovine serum albumin loaded poly (D, L-lactic-co-glycolic acid) microspheres by a modified phase separation technique. J Microencapsul, 2005, 22: 117-126

21 Manoharan C, Singh J. Insulin loaded PLGA microspheres: effect of zinc salts on encapsulation, release, and stability. J Pharm Sci, 2009, 98: 529-542

22 Hinds K D, Campbell K M, Holland K M, et al. PEGylated insulin in PLGA microparticles. In vivo and in vitro analysis. J Controlled Release, 2005, 104: 447-460

23 Wang B, Song Y, Wang F, et al. Effects of local infiltration of insulin around titanium implants in diabetic rats. Br J Oral Maxillofac Surg, 2010, 49: 225-229

24 Wang F, Song Y L, Li C X, et al. Sustained release of insulin-like growth factor-1 from poly(lactide-co-glycolide) microspheres improves osseointegration of dental implants in type 2 diabetic rats. Eur J Pharmacol, 2010, 640: 226-232

25 Kaufman M R, Westreich R, Ammar S M, et al. Autologous cartilage grafts enhanced by a novel transplant medium using fibrin sealant and fibroblast growth factor. Arch Facial Plast Surg, 2004, 6: 94-100

26 Gwak S J, Kim S S, Sung K, et al. Synergistic effect of keratinocyte transplantation and epidermal growth factor delivery on epidermal regeneration. Cell Transplant, 2005, 14: 809-817

27 Spicer P P, Mikos A G. Fibrin glue as a drug delivery system. J Controlled Release, 2010, 148: 49-55

28 Jeon O, Kang S W, Lim H W, et al. Long-term and zero-order release of basic fibroblast growth factor from heparin-conjugated poly(Llactide-co-glycolide) nanospheres and fibrin gel. Biomaterials, 2006, 27: $1598-1607$
29 Zhou W, Zhao M, Zhao Y, et al. A fibrin gel loaded with chitosan nanoparticles for local delivery of rhEGF: preparation and in vitro release studies. J Mater Sci Mater Med, 2011, 22: 1221-1230

30 Lauterbach E C. Dextromethorphan as a potential rapid-acting antidepressant. Med Hypotheses, 2011, 76: 717-719

31 Zhang $\mathrm{L}$, $\mathrm{Li} \mathrm{H}$, $\mathrm{Hu} \mathrm{X}$, et al. Glucocorticoid-induced p11 over-expression and chromatin remodeling: A novel molecular mechanism of traumatic stress? Med Hypotheses, 2011, 76: 774-777

32 Hill D J, Milner R D. Insulin as a growth factor. Pediatr Res, 1985, 19: 879-886

33 Sinha V R, Trehan A. Biodegradable microspheres for protein delivery. J Controlled Release, 2003, 90: 261-280

34 Branemark P I, Hansson B O, Adell R, et al. Osseointegrated implants in the treatment of the edentulous jaw. Experience from a 10-year period. Scand J Plast Reconstr Surg Suppl, 1977, 16: 1-132

35 Terada M, Inaba M, Yano Y, et al. Growth-inhibitory effect of a high glucose concentration on osteoblast-like cells. Bone, 1998, 22: 17-23

36 Zhen D, Chen Y, Tang X. Metformin reverses the deleterious effects of high glucose on osteoblast function. J Diabetes Complicat, 2010, 24: 334-344

37 Wang W, Zhang $\mathrm{X}$, Zheng $\mathrm{J}$, et al. High glucose stimulates adipogenic and inhibits osteogenic differentiation in MG-63 cells through cAMP/protein kinase A/extracellular signal-regulated kinase pathway. Mol Cell Biochem, 2010, 338: 115-122

38 Hrynyk M, Martins-Green M, Barron A E, et al. Sustained prolonged topical delivery of bioactive human insulin for potential treatment of cutaneous wounds. Int J Pharm, 2010, 398: 146-154

39 Shao P G, Bailey L C. Stabilization of $\mathrm{pH}$-induced degradation of porcine insulin in biodegradable polyester microspheres. Pharm Devel Technol, 1999, 4: 633-642

40 Lian J B, Stein G S. Development of the osteoblast phenotype: molecular mechanisms mediating osteoblast growth and differentiation. Iowa Orthop J, 1995, 15: 118-140

Open Access This article is distributed under the terms of the Creative Commons Attribution License which permits any use, distribution, and reproduction in any medium, provided the original author(s) and source are credited. 